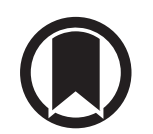

CrossMark

\title{
Transcriptomic gene signatures associated with persistent airflow limitation in patients with severe asthma
}

\author{
Pieter-Paul Hekking ${ }^{1}$, Matthew J. Loza ${ }^{2}$, Stelios Pavlidis ${ }^{3}$, Bertrand De \\ Meulder ${ }^{4}$, Diane Lefaudeux ${ }^{4}$, Frederic Baribaud ${ }^{2}$, Charles Auffray ${ }^{4}$, Ariane \\ H. Wagener ${ }^{1}$, Paul Brinkman ${ }^{1}$, René Lutter ${ }^{1}$, Aruna T. Bansal ${ }^{5}$, Ana R. Sousa ${ }^{6}$, \\ Stewart A. Bates ${ }^{6}$, Ioannis Pandis ${ }^{3}$, Louise J. Fleming ${ }^{3}$, Dominick E. Shaw ${ }^{7}$, \\ Stephen J. Fowler ${ }^{8}$, Yike Guo ${ }^{3}$, Andrea Meiser ${ }^{3}$, Kai Sun $^{3}$, Julie Corfield ${ }^{9}$, \\ Peter Howarth ${ }^{10}$, Elisabeth H. Bel ${ }^{1}$, Ian M. Adcock ${ }^{11}$, Kian Fan Chung ${ }^{11}$, \\ Ratko Djukanovic ${ }^{10}$ Peter J. Sterk ${ }^{1}$ and the U-BIOPRED Study Group ${ }^{12}$
}

Affiliations: ${ }^{1}$ Dept of Respiratory Medicine, Academic Medical Center, Amsterdam, The Netherlands. ${ }^{2}$ Janssen R\&D, Johnson \& Johnson, Springhouse, PA, USA. ${ }^{3}$ Data Science Institute, Imperial College London, London, UK. ${ }^{4}$ European Institute for Systems Biology and Medicine, CIRI UMR5308, CNRS-ENS-UCBL-INSERM, Université de Lyon, Lyon, France. ${ }^{5}$ Acclarogen, Cambridge, UK. ${ }^{6}$ Discovery Medicine, GlaxoSmithKline, Brentford, UK. ${ }^{7}$ Respiratory Research Unit, University of Nottingham, Nottingham, UK. ${ }^{8}$ Centre for Respiratory Medicine and Allergy, Institute of Inflammation and Repair, University of Manchester and University Hospital of South Manchester, Manchester Academic Health Sciences Centre, Manchester, UK. ${ }^{9}$ Areteva, Nottingham, UK. ${ }^{10}$ NIHR Southampton Centre for Biomedical Research, University of Southampton, Southampton, UK.

${ }^{11}$ National Heart and Lung Institute, Imperial College London, London, UK. ${ }^{12}$ A full list of the U-BIOPRED Study Group members and their affiliations can be found in the supplementary material.

Correspondence: Pieter-Paul Hekking, Dept of Respiratory Medicine, F5-260, Academic Medical Center, Meibergdreef 9, 1105 AZ Amsterdam, The Netherlands. E-mail: p.w.hekkingdamc.uva.nl

@ERSpublications

Persistent airflow limitation in severe asthma is associated with a mechanism involving IL-13 and remodelling http://ow.ly/JYcC30daSRf

Cite this article as: Hekking PP, Loza MJ, Pavlidis S, et al. Transcriptomic gene signatures associated with persistent airflow limitation in patients with severe asthma. Eur Respir J 2017; 50: 1602298 [https://doi.org/ 10.1183/13993003.02298-2016].

ABSTRACT A proportion of severe asthma patients suffers from persistent airflow limitation (PAL), often associated with more symptoms and exacerbations. Little is known about the underlying mechanisms. Here, our aim was to discover unexplored potential mechanisms using Gene Set Variation Analysis (GSVA), a sensitive technique that can detect underlying pathways in heterogeneous samples.

Severe asthma patients from the U-BIOPRED cohort with PAL (post-bronchodilator forced expiratory volume in $1 \mathrm{~s}$ /forced vital capacity ratio below the lower limit of normal) were compared with those without PAL. Gene expression was assessed on the total RNA of sputum cells, nasal brushings, and endobronchial brushings and biopsies. GSVA was applied to identify differentially enriched predefined gene signatures based on all available gene expression publications and data on airways disease.

Differentially enriched gene signatures were identified in nasal brushings ( $n=1)$, sputum ( $n=9)$, bronchial brushings $(n=1)$ and bronchial biopsies $(n=4)$ that were associated with response to inhaled steroids, eosinophils, interleukin-13, interferon- $\alpha$, specific $\mathrm{CD} 4^{+} \mathrm{T}$-cells and airway remodelling.

PAL in severe asthma has distinguishable underlying gene networks that are associated with treatment, inflammatory pathways and airway remodelling. These findings point towards targets for the therapy of PAL in severe asthma. 


\section{Introduction}

A small proportion of asthma patients suffers from uncontrolled disease despite treatment with high-dose inhaled or oral steroids, which is defined as severe asthma $[1,2]$. These patients are in need of effective and steroid-sparing treatments. However, severe asthma is a heterogeneous disease, which complicates the development of these treatments [3, 4]. Emerging targeted treatments appear to be successful in selected subgroups of patients with specific characteristics, such as eosinophilic airway inflammation and allergy, emphasising the need for precision medicine in severe asthma [5-7].

Agusti et al. [8] recently described a novel strategy for the treatment of chronic airways disease, focusing on treatable traits based on clinical, physiological and/or biological characteristics rather than solely on a diagnosis of asthma or chronic obstructive pulmonary disease (COPD). Persistent airflow limitation (PAL) was postulated as a treatable trait of airways disease, being characterised by reduced expiratory flow even after the administration of a short-acting bronchodilator. Notably, PAL is not only a determinant for the diagnosis of COPD, but regardless of smoking history, has also long been implicated in patients with severe asthma [9].

PAL is clinically relevant in severe asthma as it may have prognostic implications. Even though PAL and exacerbations are different phenotypic characteristics, there is evidence of an association of PAL with more frequent exacerbations and more hospitalisations in subgroups of severe asthma patients [10, 11]. Clinical clustering studies showed that PAL is an important constituent for severe asthma phenotypes that are characterised by eosinophilic airway inflammation, poor quality of life and smoking history [11-14]. To understand this clinical trait, several studies have been conducted to identify risk factors for PAL in smoking and nonsmoking severe asthmatic subjects. Eosinophilic airway inflammation, longer duration of disease, adult onset of disease and a history of smoking appear to be the most important factors [9, 15, 16]. However, management strategies for the prevention or treatment of PAL in severe asthma are lacking, which is unsurprising given the lack of knowledge regarding its mechanism.

Airway remodelling, associated with chronic inflammation, may contribute to PAL in asthma. Thickening of the airway smooth muscle layer, changes in extracellular matrix such as subepithelial fibrosis and infiltration of inflammatory cells in the bronchial airway wall have been linked to increased lung function decline $[15,17,18]$. Even though studies have been conducted to identify underlying pathways of airway remodelling, little is known about the underlying mechanism of PAL in severe asthma [19, 20]. Notably, there are differences in airway remodelling between patients with severe and patients with nonsevere asthma, demonstrating the need to examine the underlying pathways of PAL in patients with severe asthma themselves [21].

Gene Set Variation Analysis (GSVA) is a sensitive statistical technique that can be used for identifying underlying pathways in heterogeneous samples [22]. Instead of more conventional analysis of single-gene expression, GSVA allows between-groups comparisons of the expression of gene sets that are known to be associated with biological networks of relevance. This identifies differences in underlying pathways in severe asthma patients with PAL compared with those without PAL.

We hypothesised that patients with severe asthma and PAL exhibit different gene expression profiles compared with those without PAL. The aim of the present study was to identify transcriptomic pathways of PAL in severe asthma by applying GSVA in sputum cells, nasal brushings, and endobronchial brushings and biopsies. This study was conducted in the severe asthma adult cohort of the U-BIOPRED study, which according to a recent cluster analysis based on clinical and physiological parameters includes subsets of patients with and without PAL [23].

\section{Methods}

Detailed methods are provided in the supplementary material.

\section{Design and subjects}

This was a cross-sectional, observational study using data from the severe asthma adult cohort of U-BIOPRED. Details on the design of U-BIOPRED have been published previously [24]. Severe asthma

This article has supplementary material available from erj.ersjournals.com

Received: Nov 232016 | Accepted after revision: June 262017

Support statement: U-BIOPRED is funded by IMI. Funding information for this article has been deposited with the Crossref Funder Registry.

Conflict of interest: Disclosures can be found alongside this article at erj.ersjournals.com 
was defined according to the Innovative Medicine Initiative consensus statement [25]. Patients were included who had a confirmed diagnosis of asthma that was uncontrolled despite high-intensity asthma treatment $(\geqslant 1000 \mu \mathrm{g}$ fluticasone equivalent plus a second controller) or could only be controlled with treatment by systemic oral corticosteroids or omalizumab, including current smokers, ex-smokers and never-smokers. The U-BIOPRED study was registered at ClinicalTrials.gov with identifier NCT01976767 and was approved by the Medical Ethics Boards of all participating centres. All patients provided written informed consent.

\section{Persistent airflow limitation}

PAL was defined as post-bronchodilator forced expiratory volume in $1 \mathrm{~s}$ (FEV1)/forced vital capacity (FVC) ratio below the lower limit of normal (LLN). The LLN was calculated according to formulae provided by QuANJER et al. [26] (for male subjects: $-0.18^{\star} \mathrm{AGE}+75.41$; for female subjects: $-0.19^{\star} \mathrm{AGE}$ +78.4), consistent with the LLN defined by the Global Lung Function Initiative (GLI) [27]. In order to examine consistency with other criteria for PAL we additionally performed an analysis based on PAL defined by post-bronchodilator FEV $1<\mathrm{LLN}$.

\section{Measurements of mRNA samples}

Gene expression was assessed on the total RNA in unselected samples of severe asthma patients in the following four airway compartments: 1) nasal brushings $(n=37), 2)$ induced sputum $(n=79), 3)$ endobronchial brushings $(n=62)$ and 4) endobronchial biopsies $(n=50)$. There was no complete overlap between the samples, mainly because of limited sputum induction success rate (figure 1). For details on sample collection, see the supplementary material.

Microarray analysis was performed with the HT HG-U133+ PM microarray platform (Affymetrix, Santa Clara, CA, USA). For details, see the supplementary material.

\section{Statistical analysis}

For details on the statistical analysis on clinical variables, see the supplementary material.

The GSVA technique allows sensitive identification of differences in expression of predefined sets of genes between heterogeneous groups and can be used to explore underlying pathways [22]. Sets of genes used were based on all available gene expression publications and data on airways disease, including human and murine models, from both in vivo and in vitro studies, to ensure sensitive pathway detection. These contained studies on the gene expression associated with 1) airways disease treatment, 2) immunological pathways and 3) induced lung injury or inflammation. The latter included gene signatures that were identified at several time-points after the admission of either polyinosinic-polycytidylic acid (poly(I:C)) as a model for exacerbations [28] or bleomycin, which is used as a model for mimicking the course of fibrotic processes in the lung [29]. For this discovery study, 105 predefined gene sets were entered into the statistical models (supplementary table E8).

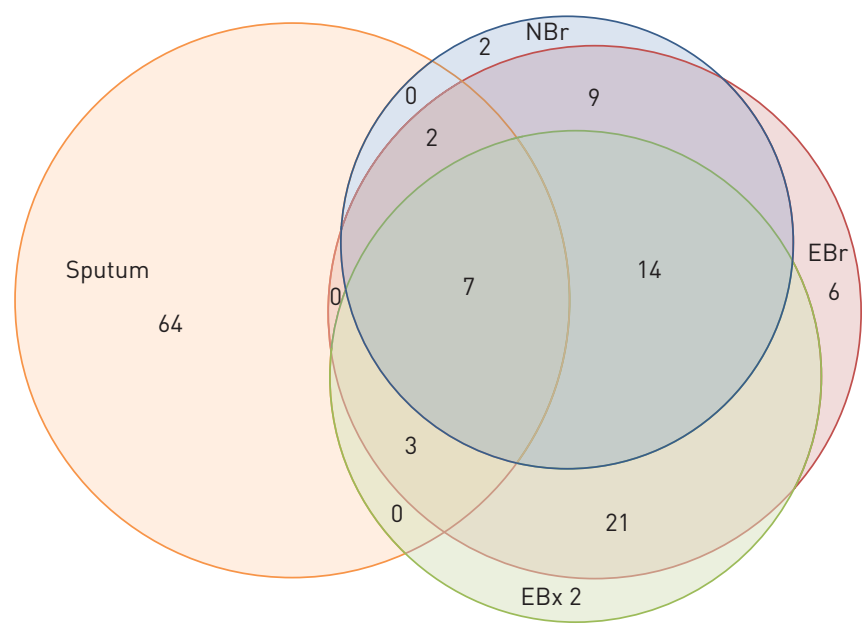

FIGURE 1 Venn diagram indicating the overlap between the samples: nasal brushings ( $\mathrm{NBr}$ ) $\mathrm{n}=37$, sputum $n=79$, endobronchial brushings $(E B r) n=62$ and endobronchial biopsies $(E B x) n=50$. Overlap of $N B r / E B x n=0$; overlap of sputum/EBx/NBr $n=3$. 
Enrichment scores and false discovery

GSVA was used to calculate the enrichment score (ES) for each patient and for each of the gene signatures based on the gene expression of genes in the sets. ES values range from -1 to 1 [22]. Subsequently, mean ES values were calculated for patients with and without PAL, and Generalised Linear Models, including correction for smoking status, corticosteroid usage and duration of asthma, were applied to statistically compare ES values between the groups. To minimise the false discovery rate, only gene signatures that had $\mathrm{p}<0.05$ and a difference of ES ( $\mathrm{dES}$ ) between the groups of $\geqslant 0.2$ were considered significantly different, following the Microarray Consortium for Quality Control (MACQC) recommendations regarding the need for applying group-difference thresholds in order to stringently limit false discovery [30].

\section{Results}

Of 421 severe asthma patients in the U-BIOPRED cohort, 224 (58\%) had PAL. These patients were significantly older and had longer asthma duration than severe asthma patients without PAL (table 1). In addition, they had significantly more eosinophilia in blood and sputum. Patients with and without PAL did not significantly differ in smoking behaviour, number of pack-years and exacerbation rate. Some distinct patient characteristics were found when patients were grouped according to site of mRNA collection. Significantly fewer females and higher blood neutrophil counts were observed in patients with PAL in the subsets of nasal and endobronchial brushings. In addition, the number of pack-years was higher in patients with PAL in the subsets of endobronchial brushings and biopsies (table 2 and see supplementary tables E1-E4 for p-values).

\section{Identified gene signatures}

In total, GSVA identified 14 unique signatures that were significantly differentially enriched in patients with PAL compared with those without PAL: $n=1$ in the nasal brushings, $n=9$ in sputum samples, $n=1$ in endobronchial brushings and $n=4$ in endobronchial biopsies (table 3 and figures 2-5).

\section{Treatment gene signatures}

Two gene signatures that were expressed to a lesser extent in asthmatic and COPD patients after treatment with fluticasone were significantly differentially enriched. The gene signature lowly expressed in fluticasone-treated asthma patients (supplementary table E7, signature 12) was significantly more enriched in the bronchial brushings of patients with PAL $(\mathrm{dES}=0.22 ; \mathrm{p}=0.0012)$. Similarly, the lowly expressed gene signature after fluticasone treatment in COPD patients, containing a different set of genes (supplementary table E7, signature 34$)$, was also enriched to a greater extent in patients with PAL $(\mathrm{dES}=0.22 ; \mathrm{p}=0.045)$ (table 3 , and figures 4 and 5).

\section{TABLE 1 Characteristics of patients with persistent airflow limitation (PAL)}

\begin{tabular}{|c|c|c|c|}
\hline & No PAL & PAL & p-value \\
\hline Patients & 197 & 224 & \\
\hline Female & $131(66.5)$ & $130(58.0)$ & 0.087 \\
\hline Age years & $49.6 \pm 14.6$ & $54.0 \pm 12.0$ & $0.001 *$ \\
\hline BMI $\mathrm{kg} \cdot \mathrm{m}^{-2}$ & $29.8 \pm 6.5$ & $28.7 \pm 6.0$ & 0.081 \\
\hline Asthma duration years & $20.0(10.0-31.0)$ & $25.5(13.0-41.0)$ & $0.001 *$ \\
\hline Smoking history & & & 0.084 \\
\hline Never-smoker & $133(67.5)$ & $131(58.5)$ & \\
\hline Ex-smoker & $50(25.4)$ & $65(29.0)$ & \\
\hline Current smoker & $14(7.1)$ & $28(12.5)$ & \\
\hline Pack-years & $9.4(3.9-20.0)$ & $13.0(4.5-22.5)$ & 0.407 \\
\hline OCS dose $\mathrm{mg}$ & $10.0(8.0-20.0)$ & $10.0(7.0-15.0)$ & 0.288 \\
\hline ACQ score & $2.6 \pm 1.2$ & $2.7 \pm 1.2$ & 0.143 \\
\hline 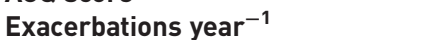 & $2.0(2.0-4.0)$ & $2.0(1.3-4.0)$ & 0.750 \\
\hline Post-bronchodilator FEV 1 \% pred & $89.0 \pm 16.1$ & $63.7 \pm 18.0$ & $<0.001 *$ \\
\hline Blood eosinophils $\times 10^{9} \mathrm{~L}^{-1}$ & $0.2(0.1-0.4)$ & $0.3(0.1-0.5)$ & $0.009^{*}$ \\
\hline Blood neutrophils $\times 10^{9} \mathrm{~L}^{-1}$ & $4.7(3.7-6.2)$ & $5.0(3.7-7.5)$ & 0.119 \\
\hline Sputum eosinophils \% & $1.2(0.2-4.6)$ & $4.8(1.2-22.1)$ & $0.001 *$ \\
\hline Sputum neutrophils \% & $51.2 \pm 26.0$ & $54.6 \pm 26.2$ & 0.412 \\
\hline
\end{tabular}

Data are presented as $\mathrm{n}, \mathrm{n}(\%)$, mean \pm SD or median (interquartile range), unless otherwise stated. BMI: body mass index; OCS: oral corticosteroid; ACQ: Asthma Control Questionnaire; FEV1: forced expiratory volume in $1 \mathrm{~s} .{ }^{*}: \mathrm{p}<0.05$. 
TABLE 2 Characteristics of patients with persistent airflow limitation (PAL) according to site of mRNA collection

\begin{tabular}{|c|c|c|c|c|c|c|c|c|}
\hline & \multicolumn{2}{|c|}{ Nasal brushings } & \multicolumn{2}{|c|}{ Sputum samples } & \multicolumn{2}{|c|}{ Endobronchial brushings } & \multicolumn{2}{|c|}{ Endobronchial biopsies } \\
\hline & No PAL & PAL & No PAL & PAL & No PAL & PAL & No PAL & PAL \\
\hline Patients & 15 & 22 & 21 & 58 & 30 & 32 & 22 & 28 \\
\hline Female & $11(73.3)^{*}$ & $8(36.4)^{*}$ & $13(61.9)$ & $35(60.3)$ & $19(63.3)^{*}$ & $10(31.2)^{*}$ & $14(63.6)$ & $12(42.9)$ \\
\hline Age years & $47.5 \pm 16.2$ & $53.2 \pm 12.1$ & $52.5 \pm 13.9$ & $54.2 \pm 11.4$ & $46.7 \pm 14.0$ & $53.0 \pm 11.1$ & $48.4 \pm 12.6$ & $52.7 \pm 11.2$ \\
\hline $\mathrm{BMI} \mathrm{kg} \cdot \mathrm{m}^{-2}$ & $34.1 \pm 6.6$ & $29.4 \pm 5.7$ & $28.2 \pm 5.0$ & $28.1 \pm 5.3$ & $31.3 \pm 6.3$ & $28.5 \pm 6.1$ & $30.2 \pm 5.4$ & $28.6 \pm 6.4$ \\
\hline Asthma duration years & $25.0(14.0-38.0)$ & $21.5(17.0-40.5)$ & $23.1 \pm 14.5$ & $26.8 \pm 17.2$ & $26.4 \pm 16.8$ & $26.7 \pm 17.9$ & $25.8 \pm 15.5$ & $30.5 \pm 18.7$ \\
\hline \multicolumn{9}{|l|}{ Smoking status } \\
\hline Never-smoker & $12(80.0)$ & $11(50.0)$ & $11(52.4)$ & 35 (60.3) & 22 (73.3) & 15 (46.9) & 16 (72.7) & $16(57.1)$ \\
\hline Ex-smoker & $3(20.0)$ & $8(36.4)$ & $8(38.1)$ & $18(31.0)$ & $7(23.3)$ & $12(37.5)$ & $5(22.7)$ & $6(21.4)$ \\
\hline Current smoker & $0(0.0)$ & $3(13.6)$ & $2(9.5)$ & $5(8.6)$ & $1(3.3)$ & $5(15.6)$ & $1(4.5)$ & $6(21.4)$ \\
\hline Pack-years & $5.0(3.3-5.1)$ & $20.0(11.5-38.5)$ & $13.8(2.9-19.7)$ & $10.0(2.8-18.8)$ & $5.1(1.4-8.1)^{*}$ & $18.8(7.0-24.0)^{*}$ & $5.3(2.0-13.4)^{*}$ & $21.5(18.0-27.5)^{*}$ \\
\hline OCS dose mg & $10.0(8.0-25.0)$ & $10.00(8.12-13.75)$ & $10.0(8.8-12.5)$ & $10.0(6.9-13.1)$ & $10.0(10.0-21.3)$ & $10.0(6.5-16.3)$ & $10.0(7.9-10.0)$ & $10.0(6.0-15.0)$ \\
\hline ACQ score & $1.9 \pm 1.0$ & $2.2 \pm 1.2$ & $2.6 \pm 1.4$ & $2.6 \pm 1.3$ & $2.1 \pm 1.2$ & $2.4 \pm 1.0$ & $2.3(1.3)$ & $2.4(1.1)$ \\
\hline Exacerbations year $^{-1}$ & $2.5(1.0-4.0)$ & $3.0(1.0-4.0)$ & $2.0(2.0-3.5)$ & $2.0(1.5-4.0)$ & $2.0(2.0-3.5)$ & $3.0(2.0-4.0)$ & $2.0(2.0-3.3)$ & $3.0(2.0-4.0)$ \\
\hline Post-bronchodilator FEV $1 \%$ pred & $99.4 \pm 17.6 *$ & $71.5 \pm 14.9 *$ & $90.2 \pm 14.0 *$ & $63.5 \pm 19.9 *$ & $91.9 \pm 14.3^{*}$ & $66.7 \pm 15.5^{*}$ & $89.6 \pm 13.6 *$ & $68.3 \pm 15.2 *$ \\
\hline Blood eosinophils $\times 10^{9} \mathrm{~L}^{-1}$ & $0.2(0.1-0.4)$ & $0.2(0.1-0.3)$ & $0.2(0.2-0.3)$ & $0.4(0.2-0.5)$ & $0.2(0.1-0.4)$ & $0.2(0.1-0.3)$ & $0.2(0.1-0.3)$ & $0.2(0.1-0.3)$ \\
\hline Blood neutrophils $\times 10^{9} \mathrm{~L}^{-1}$ & $3.8(3.6-5.1)$ & $5.0(4.2-7.8)$ & $4.3(3.3-6.3)$ & $5.0(3.9-7.5)$ & $4.0(3.2-5.4)^{*}$ & $5.8(4.1-7.7)^{*}$ & $4.0(3.5-5.3)$ & $5.0(4.3-7.3)$ \\
\hline Sputum eosinophils \% & $1.4(0.4-4.6)$ & $2.6(0.2-13.8)$ & $1.3(0.2-3.8)^{*}$ & $6.2(1.3-28.5)^{*}$ & $1.4(0.6-4.6)$ & $4.5(0.2-24.9)$ & $2.0(0.7-8.0)$ & $2.6(0.2-6.1)$ \\
\hline Sputum neutrophils \% & $46.6 \pm 18.4$ & $63.2 \pm 18.8$ & $61.5 \pm 23.6$ & $58.5 \pm 27.1$ & $48.5 \pm 20.7$ & $61.6 \pm 23.3$ & $50.4 \pm 19.5$ & $56.5 \pm 27.0$ \\
\hline
\end{tabular}

Data are presented as $\mathrm{n}, \mathrm{n}(\%)$, mean \pm SD or median (interquartile range). BMI: body mass index; OCS: oral corticosteroid; ACQ: Asthma Control Questionnaire; FEV1: forced expiratory volume in $1 \mathrm{~s} .{ }^{*}: \mathrm{p}<0.05$ (significantly different between the groups within each sample). 
TABLE 3 Differentially enriched gene signatures in severe asthma patients with persistent airflow limitation (PAL) compared with those without PAL

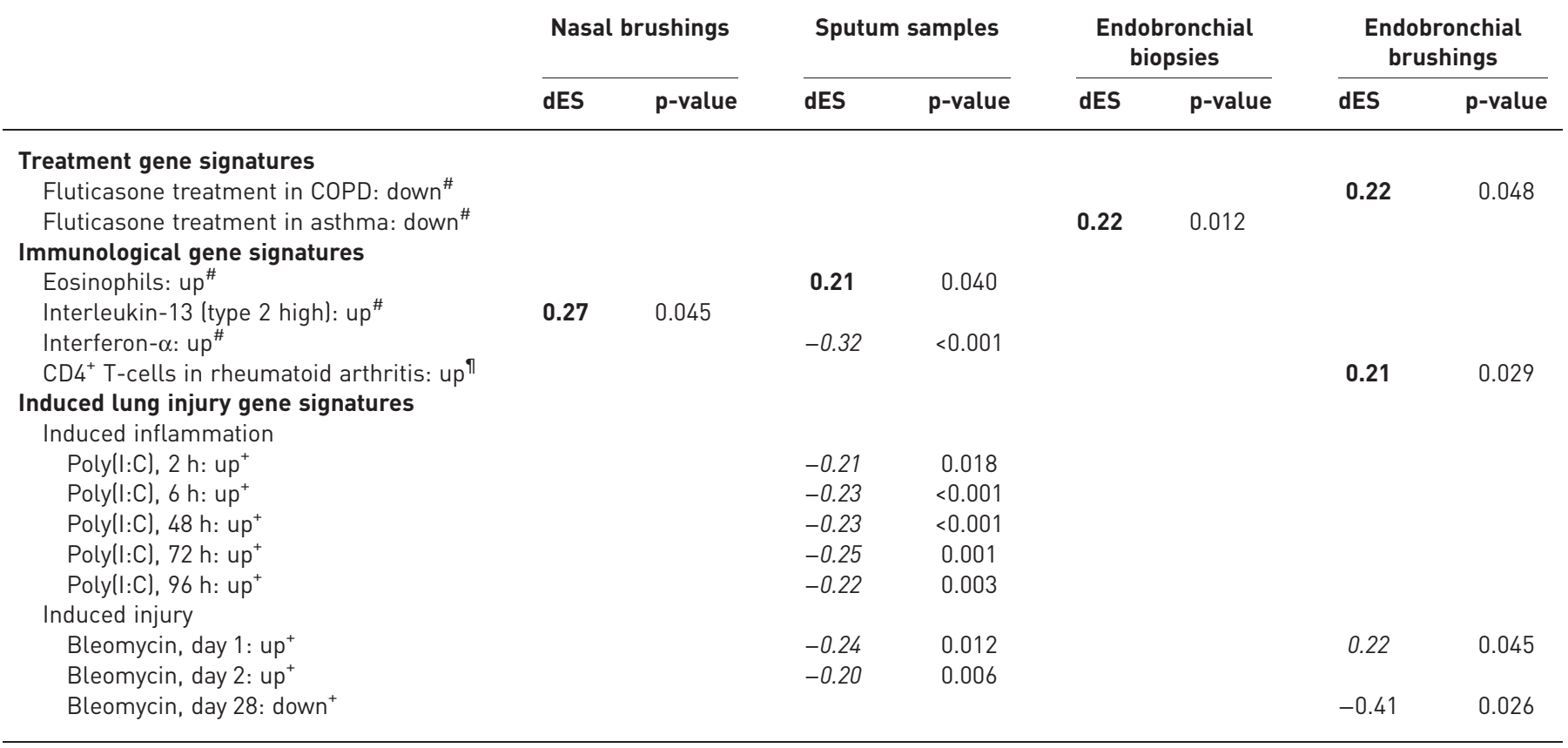

Data are presented as differences in mean gene signature enrichment score (dES) between severe asthma patients with PAL compared with those without PAL (bold indicates higher; italic indicates lower), unless otherwise stated. COPD: chronic obstructive pulmonary disease; poly(l:C): polyinosinic-polycytidylic acid. " ${ }^{\#}$ : in vitro model in human sample; ${ }^{\text {ๆ }}$ : in vivo model in human sample; ${ }^{+}$: in vivo model in murine model.

\section{Immunological gene signatures}

Four gene signatures that are associated with immunological pathways were identified to be significantly differentially enriched between the groups. 1) A gene signature that is upregulated in the presence of eosinophils (supplementary table E7, signature 55) was more enriched in the sputum of patients with PAL compared with those without PAL ( $\mathrm{dES}=0.21 ; \mathrm{p}=0.04$ ) (figure 3). 2) A gene signature that is identified to be associated with the presence of interleukin (IL)-13 (supplementary table E7, signature 101) was more enriched in the nasal brushings of PAL patients ( $\mathrm{dES}=0.27 ; \mathrm{p}=0.045$ ) (figure 2). 3) The ES for the interferon (IFN)- $\alpha$ gene signature (supplementary table E7, signature 36) was less enriched in the sputum of patients with PAL ( $\mathrm{dES}=0.32$; $\mathrm{p}<0.001$ ) (figure 3). 4) The ES for $\mathrm{CD}^{+}{ }^{+} \mathrm{T}$-cells of rheumatoid arthritis patients (supplementary table E7, signature 27) was more enriched in patients with PAL ( $\mathrm{dES}=0.21 ; \mathrm{p}=0.029)$ (figure 3 ).

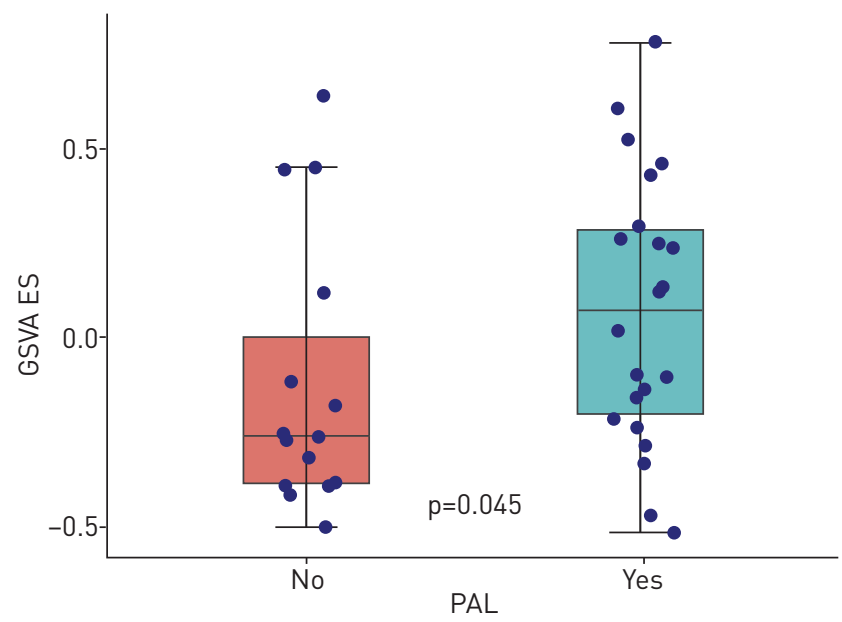

FIGURE 2 Significant gene signatures identified in nasal brushing samples. GSVA: Gene Set Variation Analysis; ES: enrichment score; PAL: persistent airflow limitation. Immunological gene signature: interleukin-13 ltype 2 high): up. 
a)

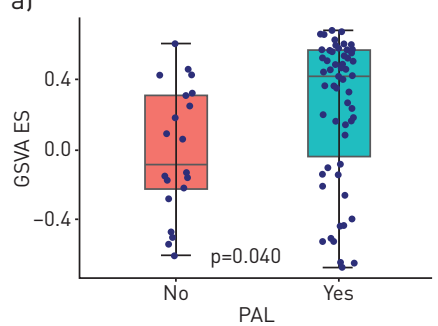

c)

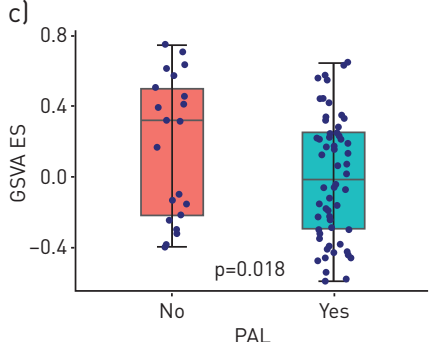

g)

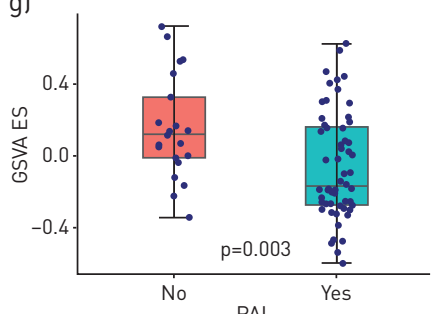

b)

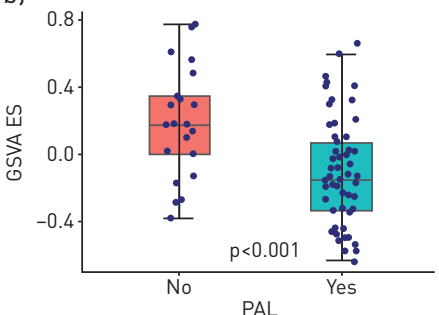

d)

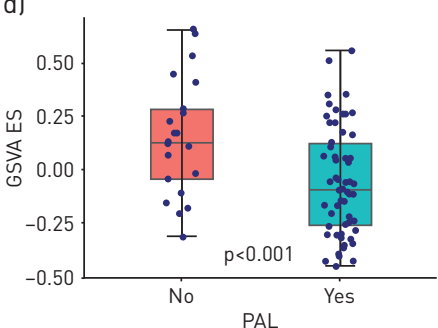

e)

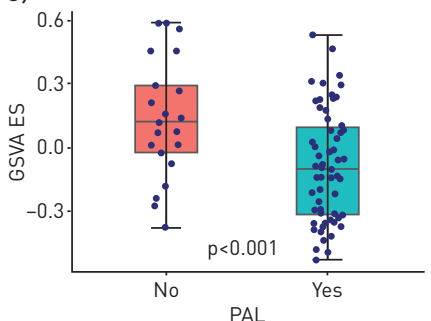

h)
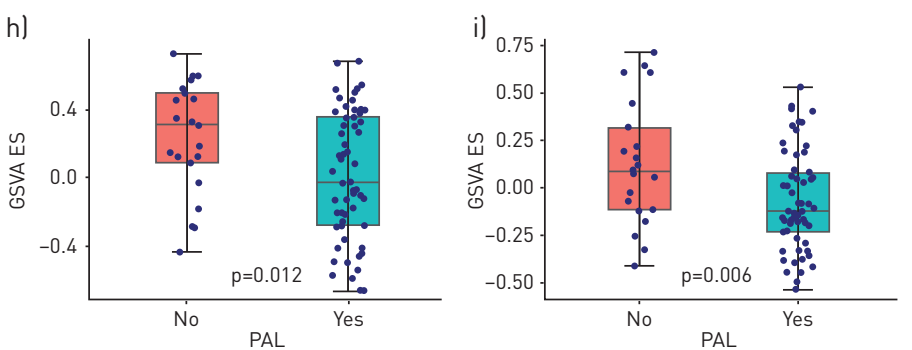

f)

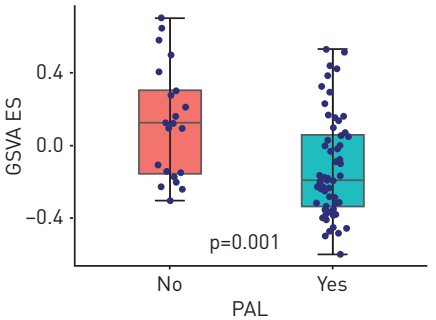

FIGURE 3 Significant gene signatures identified in sputum samples. GSVA: Gene Set Variation Analysis; ES: enrichment score; PAL: persistent airflow limitation. Immunological gene signatures: al eosinophils: up and b) interferon- $\alpha$ : up. Induced lung injury gene signatures: c) polyinosinicpolycytidylic acid (poly(l:C)), $2 \mathrm{~h}$ : up, d) poly(l:C), 6 h: up, e) poly(l:C), 48 h: up, f) poly(l:C), 72 h: up, g) poly(l:C), 96 h: up, h) bleomycin, day 1: up and i) bleomycin, day 28: down.

Induced lung injury gene signatures

In total, eight gene signatures that are associated with induced airway inflammation or lung injury were identified to be significantly differentially enriched. Five of them were discovered in a study in which gene expression was assessed at different time-points after the admission of poly(I:C) (supplementary table E7, signatures 57, 58, 62, 64 and 65), mimicking viral replication. Patients with PAL had significantly lower enrichment of these signatures in sputum, ranging from $\mathrm{dES}=-0.25$ to -0.20 (figure 3 ). In addition, three gene signatures associated with bleomycin-induced lung injury (supplementary table E7, signatures 67, 68

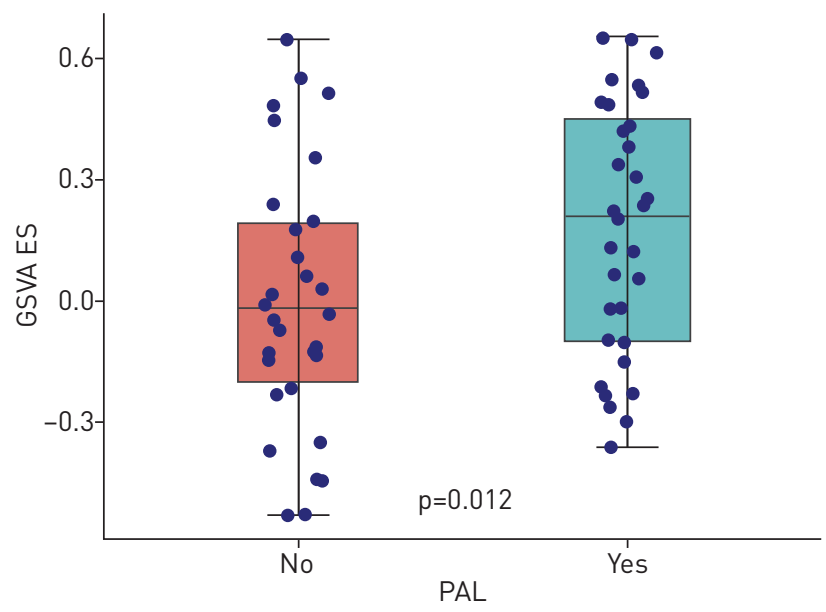

FIGURE 4 Significant gene signatures identified in endobronchial brushing samples. GSVA: Gene Set Variation Analysis; ES: enrichment score; PAL: persistent airflow limitation. Treatment gene signature: fluticasone treatment in asthma: down. 

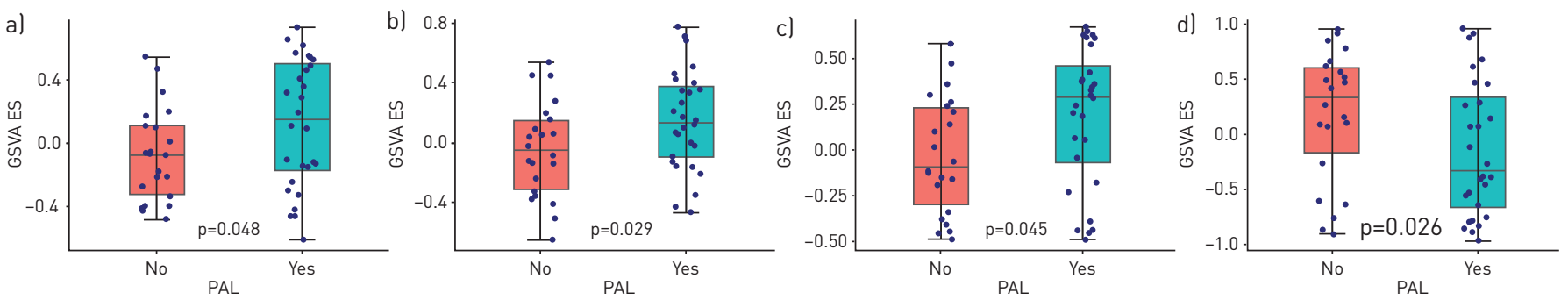

FIGURE 5 Significant gene signatures identified in endobronchial biopsy samples. GSVA: Gene Set Variation Analysis; ES: enrichment Score; PAL: persistent airflow limitation. Treatment gene signature: al fluticasone treatment in chronic obstructive pulmonary disease: down. Immunological gene signature: b) $\mathrm{CD}^{+}$T-cells in rheumatoid arthritis: up. Induced lung injury gene signatures: c) bleomycin, day 1: up and d) bleomycin, day 28: down.

and 77) were significantly differentially enriched, one associated with higher expression of genes during early development of injury (supplementary table E7, signature 67) demonstrating a significantly lower ES in sputum $(\mathrm{dES}=-0.24 ; \mathrm{p}=0.012)$ and a higher $\mathrm{ES}$ in endobronchial biopsy $(\mathrm{dES}=0.22 ; \mathrm{p}=0.045)$ in PAL patients. In addition, the gene signatures identified to be expressed higher at later stages of bleomycin-induced injury (supplementary table E7, signatures 67 and 77) had a lower ES in PAL patients $(\mathrm{dES}=-0.20 ; \mathrm{p}=0.006$ and $\mathrm{dES}=-0.41 ; \mathrm{p}=0.026)$ (table 3 and figure 5).

\section{Consistency when using different definitions of PAL}

The data when using post-bronchodilator FEV1 for defining PAL are given in the supplementary material. The data showed that, in general, signatures identified between FEV1/FVC <LLN and FEV1 <LLN were similar, including those associated with treatment with fluticasone, eosinophilic inflammation, and involvement of T-helper type 2 (Th2) cells and IFN- $\alpha$. In addition, induced lung injury and inflammation gene signatures were also identified in both analyses (supplementary table E6). However, specific involvement of interleukin (IL)-13 and $\mathrm{CD}^{+} \mathrm{T}$-cells of rheumatoid arthritis was not consistent, and no significant gene signatures were identified in the biopsies when applying FEV1 <LLN.

\section{Discussion}

This study shows that severe asthma patients with PAL have differentially enriched gene sets compared with patients without PAL, suggesting that distinguishable underlying mechanisms are involved. The identified gene signatures may indicate that these patients respond or have responded differently to treatment with inhaled corticosteroids and that particular immunological pathways are involved. These include the already recognised eosinophilic airway inflammation, but also specific $\mathrm{CD} 4^{+} \mathrm{T}$-cells and involvement of IL-13, possibly via Th2 cells. In addition, a differentially expressed gene network associated with remodelling was identified in the endobronchial airway wall, while not being identified in other compartments, underlining local involvement of remodelling. These results show that PAL is biologically distinguishable in severe asthma and point towards possible targets for the prevention and treatment of PAL.

This study is the first to assess gene signatures underlying PAL for the discovery of its mechanisms in severe asthma. Clinical characterisation of the present patients with PAL showed no association with exacerbation rate. Multiple studies in severe asthma have shown mixed results. Some found associations between more frequent exacerbations and PAL, especially in certain phenotypes of severe asthma [10, 11], whereas others observed no association [13] or even an inverse association indicative of more frequent exacerbations in patients without PAL [9]. This suggests that certain subgroups of severe asthma patients may suffer from both clinical traits. However, in a broad severe asthma population, such as the present study, PAL and exacerbations may need to be seen as separate clinical traits.

GSVA not only confirmed expected mechanisms, but also identified novel pathways in PAL. First, the results indicate that the airways of severe asthma patients with PAL respond differently to the administration of inhaled corticosteroids. Even though a relationship between severe asthma and resistance to steroids has been recognised [31], a specific association with PAL has not previously been reported. Second, not surprisingly, a gene set associated with eosinophilic airway inflammation was identified in the sputum of patients with PAL. Eosinophilic airway inflammation has been reported in patients with PAL by multiple studies in severe asthma and was also present in this study population $[9,14,15]$. Third, IL-13 was identified to be involved in PAL in severe asthma, which was in concordance with a study by KAMINSKA et al. [32] who identified IL-13 in bronchial biopsies of severe asthma patients with PAL.

Interestingly, in sputum samples, we identified a gene set associated with IFN- $\alpha$ that was less enriched in patients with PAL. Impaired or decreased IFN- $\alpha$ has been associated with severe asthma compared with 
controls $[33,34]$ and, in a murine asthma model, where IFN- $\alpha$ inhibits eosinophilic airway inflammation and hyperresponsiveness [35]. However, a direct association with PAL in (severe) asthma has not been described previously. In addition, patients with PAL appear to have lower enrichment of gene sets expressed after exposure to poly(I:C), mimicking viral replication upon infection. The recognised link between poly(I:C) and IFNs strengthens these results [36]. Impaired lung function has been found after exposure to poly(I:C) in mice [37]; however, it is unknown to what extent the ability to respond to viral infection influences lung function. Moreover, the present data indicate a role for $\mathrm{CD}_{4}^{+} \mathrm{T}$-cells, which have been associated previously with reduced lung function in patients with asthma [18]. Finally, remodelling has been recognised to be associated with PAL in (severe) asthma $[17,18]$; in concert with this, our data identified gene sets associated with bleomycin-induced lung injury and fibrotic processes in the endobronchial airway wall to be more enriched in severe asthma patients with PAL.

We believe the strengths of this study include the following. It was conducted in the severe asthma cohort of the pan-European U-BIOPRED study, which included a large number of well-selected and defined patients. The study included severe asthma patients with PAL and those without PAL with equally severe disease as an appropriate control group for analysing the distinguishing gene signatures of PAL among patients with severe asthma. The multicentre design allowed standardised collection of large numbers of samples from patients all over Europe. In addition, collection of these samples was standardised and performed according to standard operating procedures, while mRNA expression in these samples was analysed centrally to gain optimised data quality. Furthermore, using GSVA as a sensitive statistical technique enabled the identification of underlying pathways involved in PAL even in heterogeneous samples, such as in severe asthma. Finally, selection bias was minimised by correction for smoking status, asthma duration and usage of oral corticosteroids as such. However, correction for corticosteroid dose was not indicated as this was similar between the groups. Similarly, no correction for cell counts was applied as this could partly obscure the disease mechanism of PAL in severe asthma.

This study also has limitations. First, this was a cross-sectional study, whereas lung function may vary over time. Stringent selection criteria for PAL were applied to minimise possible misdiagnosis of patients, but we cannot exclude longitudinal variations in PAL. The definition of PAL was derived from the LLN on two measures of PAL based on the QUANJer et al. [26] values. Applying the GLI reference equations for the LLN to our dataset had very little influence on the distribution of patients with or without PAL, since in total only three patients included in the GSVA analyses would switch groups. Second, as shown in figure 1, there is incomplete overlap of patients from whom various samples had been collected, which was unintended but inevitable due to patient consent, feasibility and quality control (e.g. for bronchoscopies and induced sputum). This hampers comparison of the identified gene sets between the different compartments. The sample size for nasal brushings, for example, was modest. Nevertheless, concordance of identified gene signatures between the different compartments (e.g. on gene sets for fluticasone treatment and bleomycin-induced injury) and concordance in identified gene signatures by applying two definitions for PAL lend validity to the identified pathways in the different samples. Third, GSVA requires predefined gene sets to be put into the analysis. These gene signatures are acquired from the literature and are based on other studies reported thus far. Inevitably, those studies had their own flaws as well, which were not taken into account in this study. In addition, some of the gene signatures are derived from mouse model studies. These data from mouse models were purposely included, because only by broad, nonselective input of gene signatures into the analysis can novel mechanisms involved in PAL be discovered. However, it needs to be emphasised that murine models will certainly deviate from human disease and it remains to be established whether the murine signatures can be used to identify phenotypic differences in severe asthma. Furthermore, by applying predefined gene sets, we may have gene interactions that are not yet annotated and thus not identified in this study. Finally, this study did not include a separate validation set or internal validation. The sample sizes were not large enough to split into a training and validation set. However, we have applied the most stringent measures for the prevention of false discovery of gene signatures by only selecting gene signatures with a low p-value and a minimum dES, following the standardised MACQC recommendations for the importance of applying group-difference thresholds for gaining the best reproducible results [30]. In addition, the results were largely consistent when defining PAL by using FEV1 or FEV1/FVC, which contributes to the validity of the data.

Based on the present results, a few well-defined mechanisms are revealed in relation to PAL in severe asthma. First, involvement of IL-13 does not seem to be surprising. Not only because it has been directly associated with PAL previously [32], but also because it has been linked to the other identified pathways in this study. IL-13 has been associated with resistance to steroids in asthma [31,38] and is recognised as a promotor for local eosinophil recruitment in the lungs [39]. In addition, IL-13 has been identified as a direct promotor for airway remodelling [40]. Thus, IL-13 may be an important hub for the several pathways involved in PAL in severe asthma. 
Second, IFN- $\alpha$ deficiency, which may impair antiviral responses [41], has been associated with persistent asthma as such [34]. In addition, a mouse model study indicates that IFN- $\alpha$ inhibits eosinophilic inflammation [33]. The results of our study imply that only the subgroup of severe asthma patients suffering from PAL exhibits IFN- $\alpha$ deficiency or impairment, which may well be associated with lower expression of gene sets associated with poly(I:C). One could speculate that IFN- $\alpha$ deficiency could result in enhanced viral loads during an infection or exacerbation and in augmented inflammation, which in turn could result in airway wall remodelling and eventually PAL. Finally, different gene signatures appeared to be enriched in the four compartments. Gene expression related to airway remodelling was found in the more profound layers of the airway wall (biopsies), but not in the superficial layers and lumen. The opposite is true for gene signatures related to inflammatory involvement, such as eosinophilia and $\mathrm{CD} 4^{+}$ T-cells. This seems to be highly relevant, perhaps not unexpectedly implying differential pathways at different locations in the respiratory tract.

PAL has been recognised as a treatable trait in chronic airways disease [8]. In particular, severe asthma patients with PAL have high disease burden and therefore are in need of more individualised management, so-called "precision medicine". The present results suggest that patients with PAL may be less responsive to inhaled steroids. Taking into account that these patients have more symptoms and higher rates of exacerbations and hospitalisations [10], they seem to be the patients in most need for targeted medicines, such as newly developed biologicals. For instance, the involvement of IL-13 pathways in these patients may make them primary candidates for treatment with anti-IL-13.

In conclusion, severe asthma patients with PAL express different gene mechanisms compared with those without, being suggestive of specific disease mechanisms, including IL-13-associated disease pathways such as eosinophilic airway inflammation and remodelling with involvement of impaired IFN- $\alpha$ function. Therefore, these may be the patients to be selected for studies on emerging new treatments.

\section{References}

1 Chung KF, Wenzel SE, Brozek JL, et al. International ERS/ATS guidelines on definition, evaluation and treatment of severe asthma. Eur Respir J 2014; 43: 343-373.

2 Hekking PP, Wener RR, Amelink M, et al. The prevalence of severe refractory asthma. J Allergy Clin Immunol 2015; 135: 896-902.

3 Hekking PP, Bel EH. Developing and emerging clinical asthma phenotypes. J Allergy Clin Immunol Pract 2014; 2: 671-680.

4 Moore WC, Hastie AT, Li X, et al. Sputum neutrophil counts are associated with more severe asthma phenotypes using cluster analysis. J Allergy Clin Immunol 2014; 133: 1557-1563.

5 Bel EH, Wenzel SE, Thompson PJ, et al. Oral glucocorticoid-sparing effect of mepolizumab in eosinophilic asthma. N Engl J Med 2014; 371: 1189-1197.

6 Busse W, Corren J, Lanier BQ, et al. Omalizumab, anti-IgE recombinant humanized monoclonal antibody, for the treatment of severe allergic asthma. J Allergy Clin Immunol 2001; 108: 184-190.

7 Chung KF. New treatments for severe treatment-resistant asthma: targeting the right patient. Lancet Respir Med 2013; 1: 639-652.

8 Agusti A, Bel E, Thomas M, et al. Treatable traits: toward precision medicine of chronic airway diseases. Eur Respir J 2016; 47: 410-419.

9 ten Brinke A, Zwinderman AH, Sterk PJ, et al. Factors associated with persistent airflow limitation in severe asthma. Am J Respir Crit Care Med 2001; 164: 744-748.

10 Ciebiada M, Domagala M, Gorska-Ciebiada M, et al. Risk factors associated with irreversible airway obstruction in nonsmoking adult patients with severe asthma. Allergy Asthma Proc 2014; 35: 72-79.

11 Moore WC, Meyers DA, Wenzel SE, et al. Identification of asthma phenotypes using cluster analysis in the Severe Asthma Research Program. Am J Respir Crit Care Med 2010; 181: 315-323.

12 Amelink M, de Nijs SB, de Groot JC, et al. Three phenotypes of adult-onset asthma. Allergy 2013; 68: 674-680.

13 Schatz M, Hsu JW, Zeiger RS, et al. Phenotypes determined by cluster analysis in severe or difficult-to-treat asthma. J Allergy Clin Immunol 2014; 133: 1549-1556.

14 Konno S, Taniguchi N, Makita H, et al. Distinct phenotypes of cigarette smokers identified by cluster analysis of patients with severe asthma. Ann Am Thorac Soc 2015; 12: 1771-1780.

15 Bumbacea D, Campbell D, Nguyen L, et al. Parameters associated with persistent airflow obstruction in chronic severe asthma. Eur Respir J 2004; 24: 122-128.

16 Lee JH, Haselkorn T, Borish L, et al. Risk factors associated with persistent airflow limitation in severe or difficult-to-treat asthma: insights from the TENOR study. Chest 2007; 132: 1882-1889.

17 Yamauchi K, Inoue H. Airway remodeling in asthma and irreversible airflow limitation - ECM deposition in airway and possible therapy for remodeling. Allergol Int 2007; 56: 321-329.

18 den Otter I, Willems LN, van Schadewijk A, et al. Lung function decline in asthma patients with elevated bronchial CD8, CD4 and CD3 cells. Eur Respir J 2016; 48: 393-402.

19 Kwak HJ, Park DW, Seo JY, et al. The Wnt/beta-catenin signaling pathway regulates the development of airway remodeling in patients with asthma. Exp Mol Med 2015; 47: e198.

20 Perry MM, Durham AL, Austin PJ, et al. BET bromodomains regulate transforming growth factor-beta-induced proliferation and cytokine release in asthmatic airway smooth muscle. J Biol Chem 2015; 290: 9111-9121.

21 Pepe C, Foley S, Shannon J, et al. Differences in airway remodeling between subjects with severe and moderate asthma. J Allergy Clin Immunol 2005; 116: 544-549. 
22 Hanzelmann S, Castelo R, Guinney J. GSVA: gene set variation analysis for microarray and RNA-seq data. BMC Bioinformatics 2013; 14: 7.

23 Lefaudeux D, De Meulder B, Loza MJ, et al. U-BIOPRED clinical adult asthma clusters linked to a subset of sputum -omics. J Allergy Clin Immunol 2017; 139: 1797-1807

24 Shaw DE, Sousa AR, Fowler SJ, et al. Clinical and inflammatory characteristics of the European U-BIOPRED adult severe asthma cohort. Eur Respir J 2015; 46: 1308-1321.

25 Bel EH, Sousa A, Fleming L, et al. Diagnosis and definition of severe refractory asthma: an international consensus statement from the Innovative Medicine Initiative (IMI). Thorax 2011; 66: 910-917.

26 Quanjer PH, Tammeling GJ, Cotes JE, et al. Lung volumes and forced ventilatory flows. Eur Respir J 1993; 6: Suppl. 16, 5-40.

27 Quanjer PH, Stanojevic S, Cole TJ, et al. Multi-ethnic reference values for spirometry for the 3-95-yr age range: the global lung function 2012 equations. Eur Respir J 2012; 40: 1324-1343.

28 Harris P, Sridhar S, Peng R, et al. Double-stranded RNA induces molecular and inflammatory signatures that are directly relevant to COPD. Mucosal Immunol 2013; 6: 474-484.

29 Peng R, Sridhar S, Tyagi G, et al. Bleomycin induces molecular changes directly relevant to idiopathic pulmonary fibrosis: a model for "active" disease. PLoS One 2013; 8: e59348.

30 Shi L, Reid LH, Jones WD, et al. The MicroArray Quality Control (MAQC) project shows inter- and intraplatform reproducibility of gene expression measurements. Nat Biotechnol 2006; 24: 1151-1161.

31 Barnes PJ. Corticosteroid resistance in patients with asthma and chronic obstructive pulmonary disease. J Allergy Clin Immunol 2013; 131: 636-645.

32 Kaminska M, Foley S, Maghni K, et al. Airway remodeling in subjects with severe asthma with or without chronic persistent airflow obstruction. J Allergy Clin Immunol 2009; 124: 45-51.

33 Wright AK, Mistry V, Richardson M, et al. Toll-like receptor 9 dependent interferon-alpha release is impaired in severe asthma but is not associated with exacerbation frequency. Immunobiology 2015; 220: 859-864.

34 Rupani H, Martinez-Nunez RT, Dennison P, et al. Toll-like receptor 7 is reduced in severe asthma and linked to an altered microRNA profile. Am J Respir Crit Care Med 2016; 194: 26-37.

35 Kikkawa Y, Sugiyama K, Obara K, et al. Interferon-alpha inhibits airway eosinophilia and hyperresponsiveness in an animal asthma model [corrected]. Asia Pac Allergy 2012; 2: 256-263.

36 Sionov E, Mayer-Barber KD, Chang YC, et al. Type I IFN induction via poly-ICLC protects mice against cryptococcosis. PLoS Pathog 2015; 11: e1005040.

37 Stowell NC, Seideman J, Raymond HA, et al. Long-term activation of TLR3 by poly(I:C) induces inflammation and impairs lung function in mice. Respir Res 2009; 10: 43.

38 Saha SK, Berry MA, Parker D, et al. Increased sputum and bronchial biopsy IL-13 expression in severe asthma. J Allergy Clin Immunol 2008; 121: 685-691.

39 Pope SM, Brandt EB, Mishra A, et al. IL-13 induces eosinophil recruitment into the lung by an IL-5- and eotaxin-dependent mechanism. J Allergy Clin Immunol 2001; 108: 594-601.

40 Zhu Z, Homer RJ, Wang Z, et al. Pulmonary expression of interleukin-13 causes inflammation, mucus hypersecretion, subepithelial fibrosis, physiologic abnormalities, and eotaxin production. J Clin Invest 1999; 103: $779-788$

41 Sykes A, Edwards MR, Macintyre J, et al. Rhinovirus 16-induced IFN-alpha and IFN-beta are deficient in bronchoalveolar lavage cells in asthmatic patients. J Allergy Clin Immunol 2012; 129: 1506-1514. 